\title{
Acute pulmonary embolism in a patient with cesarean scar pregnancy after receiving uterine artery embolization: a case report
}

This article was published in the following Dove Press journal:

Therapeutics and Clinical Risk Management

\author{
Jian Qiu' \\ Yunfeng $\mathrm{Fu}^{2}$ \\ Xiaohong Huang' \\ Longwen Shu' \\ Jiewei $\mathrm{Xu}^{3}$ \\ Weiguo $\mathrm{Lu}^{2,4}$ \\ 'Department of Obstetrics and \\ Gynecology, Huzhou Central Hospital, \\ Huzhou, Zhejiang, ${ }^{2}$ Department of \\ Gynecologic Oncology, Women's \\ Hospital, School of Medicine, Zhejiang \\ University, Hangzhou, Zhejiang, \\ ${ }^{3}$ Department of General Surgery, \\ Huzhou Central Hospital, Huzhou, \\ Zhejiang, ${ }^{4}$ Women's Reproductive \\ Health Laboratory of Zhejiang \\ Province, Hangzhou, Zhejiang, People's \\ Republic of China
}

\begin{abstract}
Uterine artery embolization (UAE) is considered to be an effective treatment for patients with cesarean scar pregnancy, especially for those presenting uncontrollable hemorrhage. However, it can also cause some potential complications. Pulmonary embolism is a rare but fatal complication in patients treated with UAE. Here, we report a case of a woman who was diagnosed with cesarean scar pregnancy presenting with pulmonary embolism during the operation of dilation and curettage after UAE treatment. Prompt resuscitation was carried out and the patient received anticoagulant treatment immediately. During the follow-up, she recovered well without any complications. We present the clinical details and imaging findings, followed by discussions of the etiology, treatments, and prevention strategies.
\end{abstract}

Keywords: pulmonary embolism, cesarean scar pregnancy, uterine artery embolization

\section{Introduction}

Cesarean scar pregnancy (CSP), the rarest form of the ectopic pregnancies, is a direct consequence of a cesarean delivery when the subsequent pregnancy implants on the site of previous cesarean section scar. Uterine artery embolization (UAE) is considered to be a viable intervention, especially for those patients with catastrophic complication like uncontrollable hemorrhage. However, a number of complications associated with this procedure have been reported. Here, for the first time, we reported a woman who was diagnosed as CSP presenting with pulmonary embolism after receiving UAE treatment. This report was approved by the ethics committee of Huzhou Central Hospital, and a written informed consent was obtained from the patient for the case details and images to be published.

\section{Case report}

A 34-year-old woman had come to our hospital with 45 days amenorrhea and 3 days of vaginal bleeding. She had a history of cesarean section 5 years ago. The transvaginal sonography revealed empty uterine cavity and empty cervical canal with a gestational sac in the anterior myometrium of lower uterine segment (Figure 1). Anterior myometrium anterior to the gestational sac was thinned out. The laboratory test result for $\beta$-hCG revealed a level of $11,399 \mathrm{mIU} / \mathrm{mL}$.

After discussion about the possible benefits and risks of each of the treatments, she opted for the treatment of UAE and suction evacuation of the pregnancy under ultrasound guidance. So the next day, the patient's uterine artery was selectively catheterized using a Rosch hepatic catheter and was embolized using gel foam sponge
Correspondence: Weiguo Lu

Department of Gynecologic Oncology, Women's Hospital, School of Medicine, Zhejiang University, Xueshi Road \#2, Hangzhou, Zhejiang 310006, People's Republic of China

Tel $+8657 \mid 8706$ I50 I

Fax +86 57। 87061878

Email lbwg@zju.edu.cn 


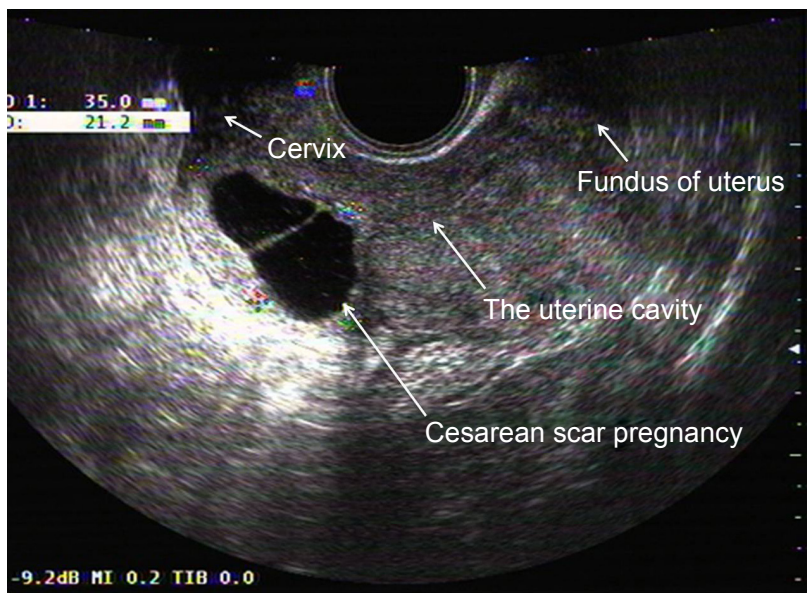

Figure I Transvaginal ultrasound scan showing empty uterine cavity and empty cervical canal with a gestational sac in anterior myometrium of lower uterine segment.

particles (900-1,200 $\mu \mathrm{m}$, Figure 2). After that, dilation and curettage were performed guided by transabdominal ultrasound. However, during the process of anesthesia, the patient presented sudden cardiac arrest. Upon rapid resuscitation (external chest compression for 8-10 seconds), she recovered immediately. After return to the ward after dilation and curettage, she presented sudden cardiac arrest once again. Rapid resuscitation (external chest compression for 8 seconds and persistent mask oxygen-inspiration was performed) was carried out one more time. The subsequent urgent bedside electrocardiography (ECG) showed abnormal Q wave in III and aVF, and the computed tomography pulmonary angiogram scan showed extensive filling defects in the pulmonary trunk artery, the left and right trunk of pulmonary arteries, upper and lower arteries of the left pulmonary lobe, and lower artery of the right pulmonary lobe (Figure 3). The next echocardiography revealed possibility of left pulmonary artery embolization, right atrium and ventricle enlargement, as well as moderate tricuspid regurgitation and mild pulmonary valve insufficiency. The patient was sent to the intensive care unit immediately, where she started to receive subcutaneous enoxaparin $100 \mathrm{IU} / \mathrm{kg}$ twice daily. A retrievable inferior vena cava filter was inserted, and the patient was kept under observation in the intensive care unit for 4 days until her condition was stabilized. After that, she was transferred to respiratory ward and remained there for 10 days. The enoxaparin was suspended, and she continued to take rivaroxaban for 2 weeks after discharge. During the next 4 months following-up, she recovered well without any complications.

\section{Discussion}

UAE is increasingly performed and considered to be an effective and minimally invasive method in treatment of
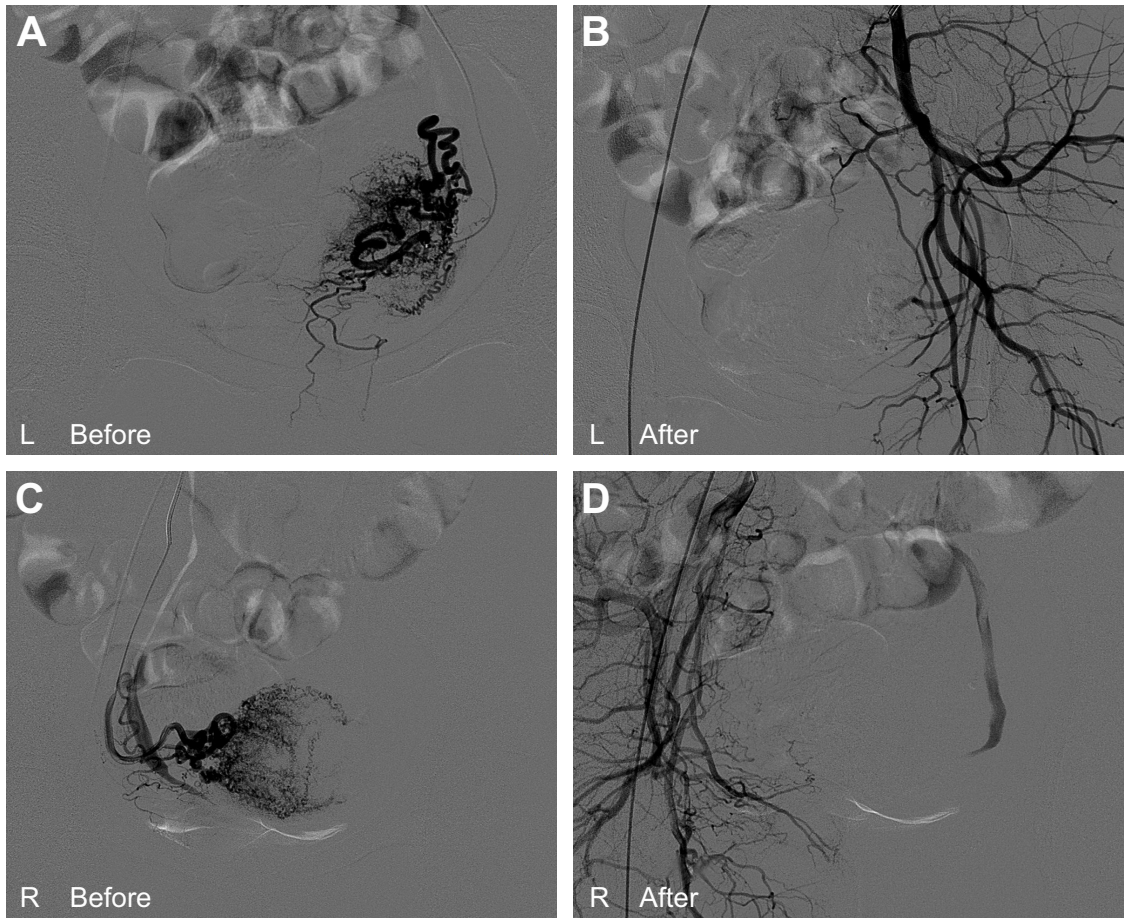

Figure 2 Digital subtraction angiogram images from the patient with CSP who was treated using transcatheter uterine arterial embolization.

Notes: (A) The left uterine artery and its branch vessels were revealed before embolization. (B) The left uterine artery and its branch vessels were not revealed after embolization. (C) The right uterine artery and its branch vessels were revealed before embolization. (D) The right uterine artery and its branch vessels were not revealed after embolization.

Abbreviation: CSP, cesarean scar pregnancy. 

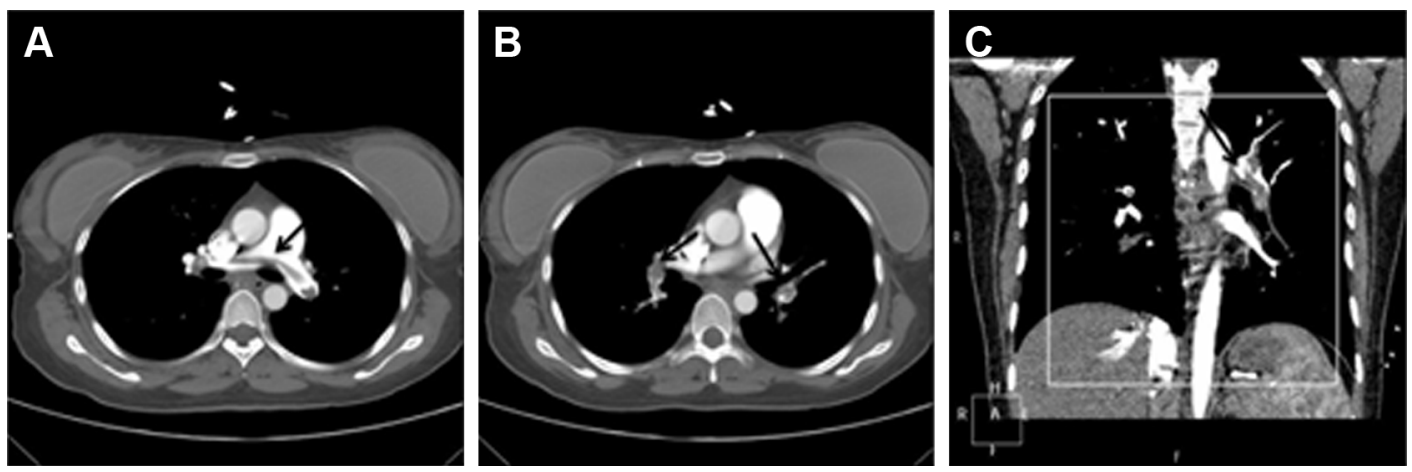

Figure 3 Computed tomography pulmonary angiogram scan showed extensive filling defects in pulmonary artery.

Notes: (A) Defects in the pulmonary trunk artery; (B) defects in the left and right trunk of pulmonary arteries; (C) defects in pulmonary trunk and branch embolization (coronary position). The arrows show the thrombus in pulmonary artery.

many obstetrical and gynecological diseases like uterine arteriovenous fistula, uterine fibroids, and, most recently, CSP. However, there are a number of known complications associated with this procedure. Major complications include infectious disease, deep venous thrombosis, ovarian dysfunction, uterine necrosis, and even death. Minor complications include hematoma, urinary tract infection, retention of urine, transient pain, and vessel or nerve injury at the puncture site. ${ }^{1,2}$ Pulmonary embolism is a rare complication of UAE $(0.25 \%$ of cases). Previously, several cases have been reported in patients with uterine fibroids who suffer from or die from pulmonary embolism after this procedure. ${ }^{3-5}$ For patients with CSP, no cases have been reported. To our knowledge, this is the first reported case in a patient with CSP who suffered from pulmonary embolism after receiving UAE treatment.

The mechanism of UAE-associated pulmonary embolism is not clear due to scarce literature. According to Virchow's theory, hypercoagulability, stasis, and injury to the vessel wall are main causes of deep venous thrombosis. ${ }^{6}$ Previous studies in patients with intracranial aneurysms or carotid artery aneurysms undergoing coil embolization show that age, number of final clips applied, number of temporary clips used, total time of temporary clip occlusion, and maximal time of temporary occlusion are risk factors for thromboembolic events. ${ }^{7,8}$ Based on Virchow's theory and previous studies, we conclude that the causes for UAE-associated pulmonary embolism may be as follows: First, the time and range for pressing on the puncture site after UAE is too long and large, which can lead to relative contrast stasis of lower-limb veins, thus producing a potential scenario for thrombosis. ${ }^{5}$ Second, disruption of the endothelial cells caused by repeated intraoperative puncture reveals a prothrombotic surface, thereby promoting coagulation and producing local thrombosis. ${ }^{9}$ Third, the embolization material, such as gelatin sponge, can fall directly into the venous system, then pass on into the arteries of the lungs, leading to pulmonary embolism. Last but most important, some patients with potential risk factors of thrombosis do not receive anticoagulant therapy immediately after UAE. As is well known, DVT is the main cause of pulmonary embolism in more than $90 \%$ of patients; pelvic veins in patients with multiple hypervascular fibroids or other gynecological disease may be enlarged and engorged, and these are prone to thrombosis owing to decreased flow after embolization. ${ }^{10}$ Other risk factors such as inherited or acquired hypercoagulability, age, using oral contraceptive agents, and historical illness associated with the risk of thrombosis like hypertension, diabetes, and hyperlipidemia may also increase the risks of thrombosis. ${ }^{6}$ In our case, due to the rarity of the complication of pulmonary embolism in patients treated with UAE, we did not check the potential risk factors of thrombosis before surgery. Considering that the patient was a young woman without any historical illness associated with the risk of thrombosis, the level of D-dimer $(0.5 \mathrm{mg} / \mathrm{L})$ before operation was not high, and the treatment of UAE is so effective that we did not consider the thromboembolism was caused by the loss of embolization agent, we suspect that the reason for pulmonary embolism in this patient after receiving UAE treatment may probably be due to the long time spent pressing on the puncture site and limb immobilization after UAE (generally, we use $1.5 \mathrm{~kg}$ sandbags on the puncture site of femoral artery for 6 hours, and the puncture limbs must be immobilized for 12 hours).

Acute pulmonary embolism is a potentially lifethreatening medical emergency and it has nonspecific signs and symptoms; thus, early recognition, as well as investigation and diagnosis are important, because early treatment with intravenous heparin and radiological interventional procedures such as vascular filters can reduce mortality. For patients with clinical suspicion of pulmonary embolism, 
examinations like ECG, computed tomography pulmonary angiogram D-dimers, or echocardiography should be taken immediately. ${ }^{11}$ Prevention of pulmonary embolism after UAE is also very important. Assessing the risk for pulmonary embolism before UAE, improving the quality of puncture so as to avoid disruption of the endothelial cells, and reducing the time of sandbag compression and limb immobilization after arterial embolization may be helpful to decrease the occurrence of pulmonary embolism. Hamoda et $\mathrm{al}^{5}$ advocated that women undergoing this procedure should routinely receive thromboprophylaxis in the form of thromboembolicdeterrent stockings. However, these would be of no benefit from an embolus originating in the pelvic veins. ${ }^{5}$ The role of using heparin-like agents in thromboprophylaxis with this procedure is also controversial because the use of such agents to decrease the incidence of embolic events may be accompanied by increased bleeding complications. ${ }^{5}$ Further research and more cases need to be investigated to determine risk factors and thromboprophylaxis for pulmonary embolism in patients receiving UAE treatment.

In summary, acute pulmonary embolism is a rare but fatal complication in patients receiving UAE treatment. Gynecologists should be aware of the possibility of such a severe adverse event during treatment with UAE, and we recommend that assessment should be proposed to determine the pulmonary embolism risk level during this procedure so as to be ready to react quickly and decisively in the operation. Once clinically suspected, necessary investigations should be arranged. Early treatment with intravenous heparin or thrombolysis is very important, as this can be life saving.

\section{Acknowledgments}

The authors would like to thank the staff of the obstetrics and gynecology ward "Guorong Yao" for providing clinical advice during the course of treatment and the staff of the intensive care unit and respiratory ward for their significant contribution to the management of our patient.

\section{Disclosure}

The authors report no conflicts of interest in this work.

\section{References}

1. Wozniakowska E, Milart P, Paszkowski T, et al. Uterine artery embolization - clinical problems. Ginekol Pol. 2013;84(12):1051-1054.

2. Kitamura Y, Ascher SM, Cooper C, et al. Imaging manifestations of complications associated with uterine artery embolization. Radiographics. 2005;25(Supp1 1):S119-S132.

3. Ojili V, Bapuraj JR, Suri V. Uterine artery embolization for the treatment of symptomatic fibroids. Int J Gynecol Obstet. 2004;87:249-251.

4. Toor SS, Jaberi A, Macdonald DB, McInnes MD, Schweitzer ME, Rasuli P. Complication rates and effectiveness of uterine artery embolization in the treatment of symptomatic leiomyomas: a systematic review and meta-analysis. AJR Am J Roentgenol. 2012;199(5):1153-1163.

5. Hamoda H, Tait P, Edmonds DK. Fatal pulmonary embolus after uterine artery fibroid embolization. Cardiovasc Intervent Radiol. 2009; 32(5):1080-1082.

6. Dalen JE. Pulmonary embolism: what have we learned since Virchow?Natural history, pathophysiology, and diagnosis. Chest. 2002;122(4): $1440-1456$.

7. Hwang G, Huh W, Lee JS, et al. Standard vs modified antiplatelet preparation for preventing thromboembolic events in patients with high on-treatment platelet reactivity undergoing coil embolization for an unruptured intracranial aneurysm: a randomized clinical trial. JAMA Neurol. 2015;72(7):764-772.

8. Namba K, Higaki A, Nemoto S. Ocular thrombosis after stentassisted coiling of a C7 (paraclinoid) internal carotid artery aneurysm. A report of two cases and literature review. Interv Neuroradiol. 2014; 20(4):455-460.

9. Elice F, Rodeghiero F, Falanga A, Rickles FR. Thrombosis associated with angiogenesis inhibitors. Best Pract Res Clin Haematol. 2009; 22(1):115-128.

10. Pakiz M, But I. Management of acute deep vein thrombosis due to enlarged symptomatic uterine fibroids. Int J Gynecol Obstet. 2009; 105(2):177-178.

11. Ryan J. Pulmonary embolism: new treatments for an old problem. Open Access Emerg Med. 2016;8:87-95.
Therapeutics and Clinical Risk Management

\section{Publish your work in this journal}

Therapeutics and Clinical Risk Management is an international, peerreviewed journal of clinical therapeutics and risk management, focusing on concise rapid reporting of clinical studies in all therapeutic areas, outcomes, safety, and programs for the effective, safe, and sustained use of medicines. This journal is indexed on PubMed Central, CAS,

\section{Dovepress}

EMBase, Scopus and the Elsevier Bibliographic databases. The manuscript management system is completely online and includes a very quick and fair peer-review system, which is all easy to use. Visit $\mathrm{http}: / /$ www.dovepress.com/testimonials.php to read real quotes from published authors. 